\title{
SHILNIKOV TYPE SOLUTIONS UNDER STRONG NON-AUTONOMOUS PERTURBATION
}

\author{
Elías Tuma
}

\begin{abstract}
We study the behaviour of solutions in a neighbourhood of the origin for a certain type of non-autonomous system of partial differential equations whose linear approximation is non autonomous.
\end{abstract}

\section{INTRODUCTION}

To study either the bifurcation which arises from a homoclinic orbit $\Gamma$, when a system of differential equations is perturbed, or the behaviour of solutions close to $\Gamma$, it is necessary to know the Poincare map defined in a transversal section of $\Gamma$, with some precision. The Poincare map is defined as a combination of two dynamics, one of them in a neighbourhood of the origin and the other in a tubular neighbourhood of the orbit $\Gamma$.

Far away from the origin we may appeal to the continuity of the solution with respect to the initial data. So we consider the solutions in a neighbourhood of the origin $[1,5]$.

This paper focuses on the derivation of exponential expansions (Deng [3], BlázquezTuma [2]) for solutions of systems of the type employed in the Shilnikov theorem [5], with a non linear, non autonomous perturbation and with a non-autonomous linear part.

Let us consider the equation

$$
\dot{z}+A z=f(t, z)
$$

where $A$ is a sectorial operator in a Banach space $X$ and $f$ is both locally Holder in $t$ and $f \in C^{k}\left(X^{\alpha}, X\right), k>2,0 \leqslant \alpha<1$, in $z$. The equation (1.1) has a local solution.

We assume that the origin is a hyperbolic equilibrium point, that is, $f(t, 0)=0$, $\forall t \in \mathbb{R}$ and the linearisation about the origin is:

$$
\dot{z}+A z=B z+C(t) z+g(t, z)
$$

where the non linear part $g(t, z)=z g_{1}(t, z)$ with $\left\|g_{1}(t, z)\right\|=0\left(\|z\|^{a}\right)$, some $a>0$, or

$$
\left\|g\left(t, z_{1}\right)-g\left(t, z_{2}\right)\right\|<k(p)\left\|z_{1}-z_{2}\right\|_{\alpha}, \quad \forall t \in \mathbb{R}
$$

Received 16th November, 2004

Financed partially by: Universidad Técnica Federico Santa María Grant No.120361.

Copyright Clearance Centre, Inc. Serial-fee code: 0004-9727/05 \$A2.00+0.00. 
with $\left\|z_{i}\right\|_{\alpha}<p, i=1,2$ and $\lim _{s \rightarrow+\infty} k(s)=0$ Let us denote by $L=A-B$ and $L(t)=A-B-C(t)$ and we assume

H1. $\sigma(L)=\sigma_{1} \bigcup \sigma_{2}$ where

(i) $\sigma_{1}=\sigma(L) \cap\{\lambda / \operatorname{Re}(\lambda)>0\}$, and there exists a simple, real eigenvalue $\beta \in \sigma_{1}$ such that $\operatorname{Re} \lambda>\beta>0, \quad \forall \lambda \in \sigma_{1}-\{\beta\}$.

(ii) $\sigma_{2}=\sigma(L) \cap\{\lambda / \operatorname{Re} \lambda<0\}$, and there exist two complex conjugate eigenvalues $\rho \pm i \omega \in \sigma_{2}$ such that $0>\rho>\operatorname{Re}(\lambda) ; \forall \lambda \in \sigma_{2}, \operatorname{Re} \lambda \neq \rho$;

(iii) $\rho+\beta>0$

Under some conditions on $C(t)$, we prove that the solution $z(t)$ of the non autonomous system (1.2) has an exponential expansion in a neighbourhood of the origin. That is, for small $\left\|z_{0}\right\|_{\alpha}=\|z(0)\|_{\alpha}$, there exist $0<\varphi<q$, such that

$$
z\left(t, t_{0}, z_{0}\right)=K\left(z_{0}, t_{0}\right) e^{-\varphi\left(t-t_{0}\right)}+\varepsilon\left(t, t_{0}\right)
$$

with $\left\|\varepsilon\left(t, t_{0}\right)\right\|_{\alpha}<C\left\|z_{0}\right\|_{\alpha} e^{-q(t-t)}$ and $K\left(z_{0}, t_{0}\right) \in \operatorname{ker}(L-\beta I)$.

The Banach space $X^{\alpha}$ can be written locally as $X^{\alpha}=X_{1}^{\alpha} \oplus X_{2}^{\alpha}$ where $X_{i}^{\alpha}, i=1,2$ are invariant sub manifolds associated to the spectral sets $\sigma_{i}$ with projections $E_{i}, i=1,2$. If $L_{i}=L / X_{i}^{\alpha}$, we have that $\sigma\left(L_{i}\right)=\sigma_{i}$ and the bounds ([4]).

$$
\begin{array}{ll}
\left\|e^{-L_{1} t} E_{1} z\right\| \leqslant M e^{-\beta t}\left\|E_{1} z\right\|_{\alpha} \leqslant M e^{-\beta t} t^{-\alpha}\left\|E_{1} z\right\| ; & t>0 \\
\left\|e^{-L_{2} t} E_{2} z\right\| \leqslant M e^{-\rho t}\left\|E_{2} z\right\|_{\alpha} \leqslant M e^{-\rho t}\left\|E_{2} z\right\| ; & t<0
\end{array}
$$

Let $x=E_{1} z$, and $y=E_{2} z$. Then equation (1.1) can be written as

$$
\begin{aligned}
& \dot{x}+L_{1}(t) x=E_{1} g(x, y, t)=g_{1}(x, y, t) \\
& \dot{y}+L_{2}(t) y=E_{2} g(x, y, t)=g_{2}(x, y, t)
\end{aligned}
$$

Let us assume either one of the following conditions on $C(t)$

H2.1 $\int_{\mathbb{R}}|C(t)|^{2} d t<P^{2}<\infty$.

$\mathrm{H} 2.2 \mathbf{C}(\mathbf{t})$ is bounded that is $\|\mathbf{C}(\mathbf{t})\|<\mathbf{k}$ some $\mathbf{k}>0$.

We know that the linear systems $\dot{x}+L_{1}(t) x=0$, and $\dot{y}+L_{2}(t) y=0$, have unique solutions $x(t)=x\left(t ; t_{0}, x_{0}\right) ; y(t)=y\left(t ; t_{0}, y_{0}\right)$ such that $x\left(t_{0}\right)=x_{0} \quad, y\left(t_{0}\right)=y_{0}$. These solution generate a family of evolution operator $\left\{T_{1}(t, s) / t>s\right\}$ and $\left\{T_{2}(t, s) / t<s\right\}$ such that

$$
x\left(t ; t_{0}, x_{0}\right)=T_{1}\left(t, t_{0}\right) x_{0} ; y\left(t ; t_{0}, y_{0}\right)=T_{2}\left(t, t_{0}\right) y_{0}
$$

Using Gronwall's inequality [4, Lemma 7.11], we obtain the following.

LEMMA 1. Under the hypothesis $\mathrm{H1}$, we have: 
(i) If $C(t)$ satisfies $\mathrm{H} 2.1$ then for $0<\alpha<1 / 2$ there exist a constant $K$ such that

$$
\begin{array}{llrl}
\left\|T_{1}(t, s)\right\|<K e^{-\beta_{1}(t-s)} ; & t>s \\
\left\|T_{2}(t, s)\right\|<K e^{-\rho(t-s)} ; & t<s
\end{array}
$$

with $\beta_{1}=\beta-P M / \sqrt{(1-2 \alpha)}>0$.

(ii) If $C(t)$ satisfies $\mathrm{H} 2.2$ then there exists a constant $K$ such that

$$
\begin{array}{ll}
\left\|T_{1}(t, s)\right\| \leqslant K e^{-\left(\beta-(k M \Gamma(1-\alpha))^{1 / 1-\alpha}\right)(t-s)} ; & t \geqslant s \\
\left\|T_{2}(t, s)\right\| \leqslant K e^{\delta(t-s)} ; & t<s
\end{array}
$$

with $\delta=\beta-k M>0$

Proof: Let us prove the bounds for $T_{1}(t, s)$ :

(i)

$$
\begin{aligned}
x(t) & =e^{-L(t-s)} x(s)+\int_{s}^{t} e^{-L(t-r)} C(r) x(r) d r\|x(t)\|_{\alpha} \\
& \leqslant M e^{-\beta(t-s)}\|x(s)\|_{\alpha}+\int_{s}^{t} M e^{-\beta(t-r)}(t-r)^{-\alpha}\|C(r)\|\|x(r)\|_{\alpha} d r \Rightarrow\|x(t)\|_{\alpha} e^{\beta(t-s)} \\
& \leqslant M\|x(s)\|_{\alpha}+\int_{s}^{t} M e^{\beta(r-s)}(t-r)^{-\alpha}\|C(r)\|\|x(r)\|_{\alpha} d r
\end{aligned}
$$

using Gronwall's inequality [4]

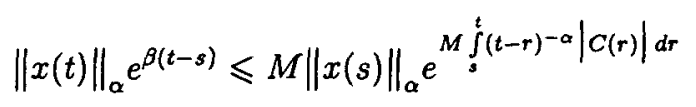

Since for $0<\alpha<1 / 2$

$$
\begin{aligned}
\int_{s}^{t}(t-r)^{-\alpha}\|C(r)\| d r & \leqslant\left(\int_{s}^{t}(t-r)^{-2 \alpha} d r\right)^{1 / 2}\left(\int_{s}^{t}\|C(r)\|^{2} d r\right)^{1 / 2} \\
& \leqslant[P / \sqrt{(1-2 \alpha)]}](t-s)^{(1 / 2-\alpha)}
\end{aligned}
$$

the result follows.

(ii)

$$
\|x(t)\|_{\alpha} \leqslant M \bar{e}^{\beta(t-s)}\|x(s)\|_{\alpha}+\int_{s}^{t} M e^{-\beta(t-r)}(t-r)^{-\alpha}\|C(r)\|\|x(r)\|_{\alpha} d r
$$


then

$$
\|x(t)\|_{\alpha} e^{\beta(t-s)} \leqslant M\|x(s)\|_{\alpha}+\int_{s}^{t} M e^{\beta(r-s)}(t-r)^{-\alpha}\|C(r)\|\|x(r)\|_{\alpha} d r
$$

Using Gronwall's inequality [4]

$$
\|x(t)\|_{\alpha} e^{\beta(t-s)} \leqslant K\|x(s)\|_{\alpha} E_{1-\alpha}(\Theta(t-s))
$$

where

$$
\Theta=(k M \Gamma(1-\alpha))^{1 / 1-\alpha} ; E_{1-\alpha}(\Theta(t-s)) \approx 1 /(1-\alpha) e^{\Theta(t-s)}
$$

Hence we have

$$
\left\|T_{1}(t, s) x(s)\right\|_{\alpha} \leqslant K\|x(s)\|_{\alpha} e^{-\left(\beta-\left(k M \Gamma(1-\alpha)^{1 / 1-\alpha}\right)(t-s)\right.} .
$$

Inmediately, from the lemma, we have.

THEOREM 1. There exist local stable $\left(W^{s}\right)$ and unstable $\left(W^{u}\right)$ manifolds of (1.4).

PROOF: Let

$$
S=\left\{z_{0} /\left\|E_{1} z_{0}\right\|<p / K,\left\|z\left(t, t_{0}, z_{0}\right)\right\|_{\alpha}\langle p, t) t_{0}\right\}
$$

If $z_{0} \in S$ then

$$
z(t)=x(t)+y(t) \in X_{1}^{\alpha} \oplus X_{2}^{\alpha},
$$

where

$$
y(t)=T_{2}\left(t, t_{0}\right) E_{2} z_{0}+\int_{t_{0}}^{t} T_{2}(t, s) E_{2} g(s, z(s)) d s
$$

Hence

$$
T_{2}(0, t) y(t)=T_{2}\left(0, t_{0}\right) E_{0} z_{0}+\int_{t_{0}}^{t} T_{2}(0, s) E_{2} g(s, z(s)) d s
$$

But

$$
\begin{aligned}
\left\|T_{2}(0, t) y(t)\right\|_{\alpha} & \leqslant K e^{-\delta t}\|y(t)\|_{\alpha} \rightarrow 0, \text { as } t \rightarrow \infty \Rightarrow T_{2}\left(0, t_{0}\right) E_{2} z_{0} \\
& =-\int_{t_{0}}^{\infty} T_{2}(0, s) E_{2} g(s, z(s)) d s \Rightarrow E_{2} z_{0} \\
& =-\int_{t_{0}}^{\infty} T_{2}(0, s) E_{2} g(s, z(s)) d s, \quad t \geqslant t_{0} \Rightarrow z(t) \\
& =T_{1}\left(t, t_{0}\right) a+\int_{t_{0}}^{t} T_{1}(t, s) E_{1} g(s, z(s)) d s-\int_{t}^{\infty} T_{2}(t, s) E_{2} g(s, z(s)) d s \unlhd R(z)
\end{aligned}
$$


say.

Similary if $a \in X_{1}$ with $\|a\|<p / 2 K$ then we shall prove that there exists a unique solution, $z\left(t, t_{0}, a\right)$, with $E_{1} z_{0}=E_{1}\left(z\left(t_{0}, t_{0}, a\right)\right)=a$, and $\|z\|_{\alpha}<p, \forall t>t_{0}$. In fact

$$
\begin{aligned}
\|z\|_{\alpha} & \leqslant K e^{-\beta_{1}\left(t-t_{0}\right)}\|a\|+\int_{t_{0}}^{t} K e^{-\beta_{1}(t-s)}\left\|E_{1} g(s, z(s))\right\| d s+\int_{t}^{\infty} K e^{\delta(t-s)}\left\|E_{2} g(s, z(s))\right\| d s \\
& \leqslant p / 2+K k(p)\left(\left\|E_{1}\right\| \int_{0}^{\infty} e^{-\beta_{1} u} d u+\left\|E_{2}\right\| \int_{0}^{\infty} e^{-\delta u} d u\right)<p
\end{aligned}
$$

so $R(z)$ is a contraction map, in the space of continuous functions with sup $\|z\|_{\alpha}<p$ and satisfying $E_{1} z\left(t_{0}\right)=a$. Hence there exist a unique fixed point $z\left(t ; t_{0}, a\right)$. Furthermore, from the integral representation it follows that the application $t \rightarrow z\left(t, t_{0}, a\right)$ is Holder continuous. Therefore if $z$ is a solution of the equation (1.2) with initial conditions.

$$
h(a) \equiv z\left(t_{0}, t_{0}, a\right)=a-\int_{t_{0}}^{\infty} T_{2}\left(t_{0}, s\right) E_{2} g(s, z(s)) d s
$$

then $E_{1} h(a) \equiv a$ Morover

$$
S=\left\{h(a) / a \in X_{1}^{\alpha},\|a\|_{\alpha} \leqslant p / 2 K\right\}
$$

and $\|h(a)-a\|_{\alpha}=0\left(\|a\|_{\alpha}\right)$. Similarly

$$
S=W^{u}(0)=\left\{h(a) / a \in X_{2}^{\alpha},\|a\|_{\alpha} \leqslant p / 2 K\right\} .
$$

REMARK. The stable and unstable manifolds are given locally by

$$
W_{\mathrm{loc}}^{s}: y=h(x, t) ; \quad W_{\mathrm{loc}}^{\mathrm{u}}: x=k(y, t)
$$

Letting

$$
x \rightarrow x-k(y, t), \quad y \rightarrow y-h(x, t)
$$

then

$$
W_{\text {loc }}^{s}: y=0 ; \quad W_{\text {loc }}^{u}: x=0
$$

and thus equation (1.4) becomes

$$
\begin{aligned}
& \dot{x}+L_{1}(t) x=f_{1}(x, y, t) x \\
& \dot{y}+L_{2}(t) y=f_{2}(x, y, t) y
\end{aligned}
$$

with $f_{i}(0,0, t)=0 \quad \forall t>0$ The integral form of $(1.5)$ is given by

$$
\begin{aligned}
& x(t)=T_{1}\left(t, t_{0}\right) x_{0}+\int_{t_{0}}^{t} T_{1}(t, s) f_{1}(s, x(s), y(s)) x(s) d s \\
& y(t)=T_{2}\left(t, t_{1}\right) y_{1}+\int_{t_{1}}^{t} T_{2}(t, s) f_{2}(s, x(s), y(s)) y(s) d s
\end{aligned}
$$


for any $t_{0}$ and $t_{1}$.

LEMMA 2. For $\left\|x_{0}\right\|_{\alpha},\left\|y_{1}\right\|_{\alpha}$ sufficiently small there exist a unique solution of (1.5) in a neighbourhood of the origin for $0<t_{0}<t_{1}$

Proof: Let

$$
H=\left\{(x, y) /\|x(t)\|_{\alpha^{\prime}}\|y(s)\|_{\alpha}<K_{1}<\infty\right\}
$$

$H$ is a complete metric space with the norm:

$$
d\left(\left(x_{1}, y_{1}\right),\left(x_{2}, y_{2}\right)\right)=\left\|x_{2}-x_{1}\right\|_{\alpha}+\left\|y_{2}-y_{1}\right\|_{\alpha}
$$

Let $T(x, y)=\left(T_{1}(x, y), T_{2}(x, y)\right)$ where $T_{1}$ and $T_{2}$ are given a the right hand side of (1.6). Then $T: H \rightarrow H$ is a contraction map:

$$
\begin{aligned}
\left\|T_{1}(x, y)\right\|_{\alpha} & \leqslant K e^{-\beta\left(t-t_{0}\right)}\left\|x_{0}\right\|_{\alpha}+\int_{t_{0}}^{t} K e^{-\beta(t-s)} k(p)\|x(s)\|_{\alpha} d s \Rightarrow\left\|T_{1}(x, y)\right\|_{\alpha} e^{\beta\left(t-t_{0}\right)} \\
& \leqslant K\left\|x_{0}\right\|_{\alpha}+K \int_{t_{0}}^{t} e^{\beta\left(s-t_{0}\right)} k(p)\|x(s)\|_{\alpha} d s \\
& \leqslant K\left\|x\left(t_{0}\right)\right\|_{\alpha}\left(1+\int_{t_{0}}^{t} k(p) d s\right) \leqslant p
\end{aligned}
$$

Similarly

$$
\left\|T_{2}(x, y)\right\|_{\alpha} \leqslant K e^{\delta\left(t-t_{1}\right)}\left\|y_{1}\right\|_{\alpha}+\int_{t}^{t_{1}} K e^{\delta(t-s)} k(p)\|y(s)\|_{\alpha} d s \leqslant p
$$

Furthermore

$$
\left\|T_{1}(x, y)-T_{1}\left(x_{1}, y_{1}\right)\right\|_{\alpha} \leqslant \int_{t_{0}}^{t} K e^{-\beta(t-s)} k(p)\left\|x(s)-x_{1}(s)\right\|_{\alpha} d s \leqslant q\left\|x-x_{1}\right\|_{\alpha}
$$

and

$$
\left\|T_{2}(x, y)-T_{2}\left(x_{1}, y_{1}\right)\right\|_{\alpha} \leqslant \int_{t}^{t_{t}} K e^{\delta(t-s)} k(p)\left\|x(s)-x_{1}(s)\right\|_{\alpha} d s \leqslant q\left\|y-y_{1}\right\|_{\alpha}
$$

with $q<1$, for small $k(p)$.

Then the result follows by the fixed point theorem.

Lemma 3. The solution of (1.1) under the hypothesis $\mathrm{H} 1-\mathrm{H} 2.1$ satisfies

$$
\|z(t)\|_{\alpha} \leqslant K_{3} e^{-\beta_{1}(t-s)}\|z(s)\|_{\alpha}
$$


Proof: Let

$$
H=\left\{z(t) /\|z(t)\|_{\alpha} \leqslant K_{3} e^{-\beta_{1}(t-s)}\|z(s)\|_{\alpha}\right\}
$$

and define on $H$

$$
F(z)=T(t, s) z(s)+\int_{s}^{t} T(t, r) g_{1}(r, z) z(r) d r
$$

Then

$$
\begin{aligned}
\|F(z)\|_{\alpha} & \leqslant K e^{-\beta_{1}(t-s)}\|z(s)\|_{\alpha}+\int_{s}^{t} K e^{-\beta_{1}(t-r)}\left\|g_{1}(r, z)\right\|\|z(r)\|_{\alpha} d r \Rightarrow\|F(z)\|_{\alpha} e^{\beta_{1}(t-s)} \\
& \leqslant K\|z(s)\|_{\alpha}+\int_{s}^{t} K K_{3} e^{\beta_{1}(r-s)}\left\|g_{1}(r, z)\right\|\|z(s)\|_{\alpha} e^{-\beta(r-s)} d r \\
& \leqslant K\|z(s)\|_{\alpha}\left(1+\int_{s}^{t} K_{3}\left\|g_{1}(r, z)\right\| d r\right) \leqslant K_{1}
\end{aligned}
$$

but

$$
\int_{s}^{t}\left\|g_{1}(r, z)\right\| d r \leqslant \int_{s}^{t} K_{3}\left(e^{-\beta_{1}(t-s)}\|z(s)\|\right)^{a} d r<K_{2}
$$

Then $F(z) \in H$ and $F$ is a contraction. In effect

$$
\|F(u)-F(v)\|_{\alpha} \leqslant \int_{s}^{t}\|T(t, r)(g(r, u)-g(r, v))\|_{\alpha} d r \leqslant \int_{s}^{t} K e^{-\beta_{1}(t-r)} k(p)\|, d r\| u-v \|_{\alpha}
$$

For small $k(p)$ we have

$$
\|F(u)-F(v)\|_{\alpha} \leqslant q\|u-v\|_{\alpha} \quad \mathrm{q}<1 .
$$

THEOREM 2. Under the conditions of the lemma the solution $z(t)$ has an exponential expansion (taking $\varphi=\beta_{1}$ ) if

$$
(2 \beta)^{1-2 \alpha}>4 P^{2} M^{2} \Gamma(1-2 \alpha)
$$

Proof: Let $X=X_{1}^{\alpha} \oplus X_{2}^{\alpha}$ where $X_{1}^{\alpha}=\operatorname{ker}(L-\beta I) ; X_{2}^{\alpha}=\Im(L-\beta I)$ and $L_{i}=L / X_{i}^{\alpha}$ and $E_{i}$ be projections, $i=1,2$. Then $z=u+v \in X^{\alpha}$. Then

$$
v(t)=e^{-L_{2}\left(t-t_{0}\right)} E_{2}\left(z_{0}\right)+\int_{t_{0}}^{t} e^{-L_{2}(t-s)}\left(C(s)+E_{2} g(t, z)\right) v(s) d s
$$

Let $\gamma, \sigma$ such that $0<\beta<\sigma<\gamma$. Then we have

$$
\|v(t)\|_{\alpha} \leqslant K e^{-\sigma\left(t-t_{0}\right)}\left\|E_{2}\left(z_{0}\right)\right\|_{\alpha} .
$$


Let

$$
H=\left\{v(t) /\|v(t)\|_{\alpha} \leqslant K_{3} e^{-\sigma\left(t-t_{0}\right)}\left\|E_{2}\left(z_{0}\right)\right\|_{\alpha}\right\}
$$

Then if $F(v)$ is the right hand side of the integral equation, we have

$$
\begin{aligned}
&\|F(v)\|_{\alpha} \leqslant M e^{-\gamma\left(t-t_{0}\right)}\left\|E_{2}\left(z_{0}\right)\right\|_{\alpha}+\int_{t_{0}}^{t} K e^{-\gamma(t-s)}(t-s)^{-\alpha}\left(\left\|E_{2} g(s, z)\right\|\right.+\|C(s)\|)\|v(s)\|_{\alpha} d s \\
& \leqslant M e^{-\gamma\left(t-t_{0}\right)}\left\|E_{0} z_{0}\right\|_{\alpha}+M e^{-\gamma\left(t-t_{0}\right)} \int_{t_{0}}^{t}(t-s)^{-\alpha}(\|C(s)\| \\
&\left.\quad+\left\|E_{2} g(s, z)\right\|\right) e^{-(\gamma-\sigma)(t-s)}\left\|E_{2} z_{0}\right\|_{\alpha} d s \\
& \leqslant M e^{-\sigma\left(t-t_{0}\right)}\left\|E_{2} z_{0}\right\|_{\alpha}\left(1+\int_{t_{0}}^{t}(t-s)^{-\alpha}\|C(s)\| e^{-(\gamma-\sigma)(t-s)} d s\right. \\
&\left.\quad+\int_{t_{0}}^{t}(t-s)^{-\alpha}\left\|E_{2} g\right\|^{-(\gamma-\sigma)(t-s)} d s\right) \\
& \leqslant e^{-\sigma\left(t-t_{0}\right)}\left\|E_{2} z_{0}\right\|_{\alpha}\left(1+\int_{t_{0}}^{t}\|C(s)\|^{2} d s\right]^{1 / 2}\left[\int_{t_{0}}^{t}(t-s)^{-2 \alpha} e^{-2(\gamma-\sigma)(t-s)} d s\right]^{1 / 2} \\
&\left.+k(p) \int_{t_{0}}^{t}(t-s)^{-\alpha} e^{-(\gamma-\sigma)(t-s)} d s\right) \leqslant K e^{-\sigma\left(t-t_{0}\right)}
\end{aligned}
$$

Furthermore $F$ is a contraction, since

$$
\begin{aligned}
\| F\left(v_{1}\right) & -F\left(v_{2}\right) \|_{\alpha} \\
& \left.\leqslant M \int_{t_{0}}^{t} e^{-\gamma(t-s)}(t-s)^{-\alpha}\left(\|C(s)\|+\left\|E_{2} g\right\|\right)\left\|v_{1}-v_{2}\right\|_{\alpha} d s\right) \\
& \leqslant M\left(\int_{t_{0}}^{t} e^{-\gamma(t-s)}(t-s)^{-\alpha}\left(\|C(s)\| d s+\int_{t_{0}}^{t} e^{-\gamma(t-s)}(t-s)^{-\alpha} k(p) d s\right)\left\|v_{1}-v_{2}\right\|_{\alpha}\right) \\
& \leqslant M\left(P\left[\int_{t_{0}}^{t} e^{-2 \gamma(t-s)}(t-s)^{-2 \alpha} d s\right]^{1 / 2}+k(p) \int_{t_{0}}^{t} e^{-\gamma(t-s)}(t-s)^{-\alpha} d s\right)\left\|v_{1}-v_{2}\right\|_{\alpha} \\
& \leqslant M\left(P\left[\frac{\Gamma(1-2 \alpha)}{(2 \gamma)^{I-2 \alpha}}\right]^{1 / 2}+L k(p)\right)\left\|v_{1}-v_{2}\right\|_{\alpha} \\
& \leqslant\left(P\left[\frac{\Gamma(1-2 \alpha)}{(2 \beta)^{I-2 \alpha}}\right]^{1 / 2}+L k(p)\right)\left\|v_{1}-v_{2}\right\|_{\alpha}
\end{aligned}
$$


So let us choose $p$ such that $M L k(p)<1 / 2$, and taking

$$
P^{2} M^{2}\left[\frac{\Gamma(1-2 \alpha)}{(2 \beta)^{1-2 \alpha}}\right]<1 / 4
$$

it follows that $\mathrm{F}$ is a contraction as required.

On the other hand

$$
\begin{aligned}
\int_{t_{0}}^{\infty}(t-s)^{-\alpha} e^{\beta_{1}\left(t-t_{0}\right)} & e^{-\beta(t-s)}\left(\|C(s)\|+\left\|E_{1} g\right\|\right)\|z(s)\|_{\alpha} d s \\
& \leqslant \int_{t_{0}}^{\infty}(t-s)^{-\alpha} e^{-\beta(t-s)} e^{\beta_{1}\left(t-t_{0}\right)}\left(\|C(s)\|+\left\|E_{1} g\right\|\right) K e^{-\beta_{1}\left(s-t_{0}\right)} E_{1} z_{0} d s \\
& \leqslant K \int_{t_{0}}^{\infty}(t-s)^{-\alpha} e^{-(P M / \sqrt{(1-2 \alpha)(t-s)}}\left(\|C(s)\|+\left\|E_{1} g\right\|\right) E_{1} z_{0} d s<\infty
\end{aligned}
$$

then

$$
\begin{aligned}
K\left(z_{0}, t_{0}\right) & =\lim _{t \rightarrow \infty} z(t) e^{\beta_{1}\left(t-t_{0}\right)} \\
& =E_{1} z_{0}+\lim _{t \rightarrow \infty} \int_{t_{0}}^{t} e^{-\beta\left(s-t_{0}\right)} e^{\beta_{1}\left(t-t_{0}\right)}\left(C(s)+E_{1} g\right) z(s) d s
\end{aligned}
$$

and $E_{2} K\left(z_{0}, t_{0}\right)=0$.

LEMMA 4. The solution of (1.1) under the hypothesis H1-H2 (b) satisfies

$$
\|z(t)\|_{\alpha} \leqslant K_{1} e^{-\left(\beta-(k M \Gamma(1-\alpha))^{1 / 1-\alpha}\left(t-t_{0}\right)\right)}\|z(t)\|_{\alpha}
$$

ProOF:

$$
\begin{aligned}
& \|z(t)\|_{\alpha} \leqslant M e^{-\beta\left(t-t_{0}\right)}\left\|z\left(t_{0}\right)\right\|_{\alpha} \\
& \quad+M \int_{s}^{t} e^{-\beta(t-s)}(t-s)^{-\alpha}\left(\|C(s)+\| g(s, u(s))\|\|_{\alpha}\right)\|z(s)\|_{\alpha} d s
\end{aligned}
$$

Then, using the inequality of $[4$, Lemma 7.11$]$, we have

$$
\|z(t)\|_{\alpha} e^{\beta\left(t-t_{0}\right)} \leqslant M\|z(t)\|_{\alpha} E_{1-\alpha}\left(\Theta\left(t-t_{0}\right)\right) \leqslant M\left\|z\left(t_{0}\right)\right\|_{\alpha} e^{-(\beta-\theta)\left(t-t_{0}\right)}
$$

where

$$
\theta=(M(k+k(p)) \Gamma(1-\alpha))^{1 / 1-\alpha}
$$

and the result follows. 
Theorem 3. Under the condition of the lemma the solution $z(t)$ has an exponential expansion taking

$$
\varphi=\beta-(k M \Gamma(1-\alpha))^{1 / 1-\alpha}>0
$$

Proof: As in Theorem 2 let us put $X^{\alpha}=X_{1}^{\alpha} \oplus X_{2}^{\alpha}, z(t)=u(t)+v(t) \in X^{\alpha}$ Then

$$
\|v(t)\|_{\alpha} \leqslant M e^{-\delta\left(t-t_{0}\right)}\left\|E_{2} z\left(t_{0}\right)\right\|_{\alpha}+M \int_{s}^{t} e^{-\delta(t-s)}(t-s)^{-\alpha}\left(\|C(s)\|+\left\|E_{2} g\right\|\right)\|v(s)\|_{\alpha} d s
$$

So that

$$
\|v(t)\|_{\alpha} e^{\delta\left(t-t_{0}\right)} \leqslant M\left\|E_{2} z\left(t_{0}\right)\right\|_{\alpha}+M \int_{t_{0}}^{t} e^{\delta\left(t-t_{0}\right)}(t-s)^{-\alpha}(k+k(p))\|v(s)\|_{\alpha} d s .
$$

Using Gronwall's inequality, we have:

$$
\|v(t)\|_{\alpha} \leqslant M\left\|E_{2} z\left(t_{0}\right)\right\|_{\alpha} e^{\left[-\delta+(M(k+k(p)) \Gamma(1-\alpha))^{1 / 1-\alpha}\right]\left(t-t_{0}\right)} .
$$

On the other hand

$$
u(t)=T\left(t, t_{0}\right) E_{1} z_{0}+\int_{t_{0}}^{t} T(t, s) E_{1} g(s, z(s)) u(s) d s,
$$

so

$$
\begin{aligned}
&\|u(t)\|_{\alpha} \leqslant K\left\|E_{1} z\left(t_{0}\right)\right\|_{\alpha} e^{-\left[\beta-(k M \Gamma(1-\alpha))^{1} / 1-\alpha\right]\left(t-t_{0}\right)} \\
&+K \int_{t_{0}}^{t} e^{-\left[\beta-(k M \Gamma(1-\alpha))^{1 / 1-\alpha}\right](t-s)}(t-s)^{-\alpha}\left\|E_{1} g\right\|\|u(s)\|_{\alpha} d s .
\end{aligned}
$$

Thus

$$
\begin{aligned}
& \|u(t)\|_{\alpha} e^{\left[\beta-(k M \Gamma(1-\alpha))^{1 / 1-\alpha}\right]}\left(t-t_{0}\right) \leqslant K\left\|E_{1} z\left(t_{0}\right)\right\|_{\alpha} \\
& \quad+K \int_{t_{0}}^{t} e^{\left[\beta-(k M \Gamma(1-\alpha))^{1 / 1-\alpha}\right]\left(s-t_{0}\right)}(t-s)^{-\alpha} e^{(-a-1)\left[\beta-(M(k+k(p)) \Gamma(1-\alpha))^{1 / 1-\alpha}\right]\left(s-t_{0}\right)} d s .
\end{aligned}
$$

Choosing $k(p)$ small enough the integral is bounded and

$$
0<\beta-\left(k M \Gamma(1-\alpha)^{1 / 1-\alpha}<\delta-\left(M(k+k(p) \Gamma(1-\alpha))^{1 / 1-\alpha}\right) .\right.
$$

Then

$$
K\left(z_{0}, t_{0}\right)=\lim _{t \rightarrow \infty} z(t) e^{-\left(\beta-(k M \Gamma(1-\alpha))^{1 / 1-\alpha}\left(t-t_{0}\right)\right.}
$$




\section{REFERENCES}

[1] M. Blázquez and E. Tuma, 'Bifurcation from homoclinic orbits to saddle-saddle point in Banach space', World Sci. Ser. Appl. Anal. 4 (1995), 91-99.

[2] M. Blázquez and E. Tuma, 'Shil'nikov's type solutions under non linear non autonomous pertubation', Proyecciones 15 (1996), 101-110.

[3] B. Deng, 'Exponential expansion with Shil'nikov's saddle focus', J. Differential Equations 82 (1989), 156-173.

[4] D. Henry, Geometric Theory of semi linear parabolic equations, Lectures Notes 840 (Springer-Verlag, Berlin, Heidelberg, New York, 1981).

[5] L.P. Shil'nikov's, 'A contribution to the problem of the structure of an extended neigbourhood of a rough equilibrium state of saddle-focus type', Math. Sbornik 10 (1970), 90-102.

Department of Mathematics

Santa Maria University

PO Box 110-V

Valparaiso

Chile

e-mail: elias.tuma@usm.cl 